\title{
Adrenergic and Noradrenergic Plasma Levels in Lesch-Nyhan Disease
}

\author{
Monique Ernst, M.D., Ph.D., Alan J. Zametkin, M.D., Daisy Pascualvaca, Ph.D., \\ John A. Matochik, Ph.D., Graeme Eisenhofer, Ph.D., Dennis L. Murphy, M.D., \\ and Robert M. Cohen, Ph.D., M.D.
}

Noradrenergic dysfunction and abnormality in monoamine oxidase (MAO) enzyme activity have been reported previously in Lesch-Nyhan (LN) disease. This study examines peripheral indices of adrenergic, noradrenergic, and MAO function in children and young adults with $L N$ disease $(\mathrm{n}=11)$, and healthy subjects $(\mathrm{n}=9)$. Blood samples, collected in identical conditions prior to a positron emission tomography (PET) study, were assayed for concentrations of epinephrine (EPI), norepinephrine (NE), and 3-methoxy-4-hydroxyphenylglycol (DHPG) (which results from the degradation of NE by monoamine oxidase type A [MAO-A]). The LN subjects had significantly higher EPI levels by $245 \%(\mathrm{p}<.00)$ and lower DHPG levels by $42 \%(\mathrm{p}<.00)$ compared to the control group. No group differences were noted in NE plasma levels. Cognitive function (IQ tested by Stanford Binet Intelligence Scale) was associated with EPI in the LN group $(\mathrm{r}=0.77, \mathrm{p}=.009)$, but not in the control group. The abnormally high EPI plasma concentrations may indicate another biochemical dysfunction secondary to the absence of the HPRT enzyme in LN patients. Such a biochemical deficit is likely to originate from the adrenal medulla, which is the primary site of EPI synthesis. The adrenal medulla may be directly affected by the absence of hypoxanthine guanine phosphoribosyl transferase (HPRT) enzyme, or may receive inappropriately high descending activation input from the brain. The abnormally low DHPG levels, in the context of normal NE levels, indicates low MAO activity, either as a primary deficit, or as secondary adaptive changes to spare NE levels that would otherwise be too low for adequate noradrenergic function.

[Neuropsychopharmacology 22:320-326, 2000]

Published by Elsevier Science Inc.
KEY WORDS: DHPG; Epinephrine; Lesch-Nyhan disease; HPRT; Self-injurious behaviour; Aggression

Lesch-Nyhan disease (LN), a rare $\mathrm{X}$-linked recessive disorder of purine synthesis, is characterized by the virtual absence of hypoxanthine guanine phosphoribosyl

From the Laboratory of Cerebral Metabolism, NIMH (ME, AJZ, JAM, RMC), Laboratory of Psychology and Psychopathology (DP), Clinical Neuroscience Branch, NINDS (GE), and Laboratory of Clinical Science, NIMH (DLM), Bethesda, MD; and the Neuroimaging Branch (ME), National Institute on Drug Abuse, Baltimore, MD.

Address correspondence to: Monique Ernst, M.D., Ph.D., Brain Imaging Center, National Institute on Drug Abuse, 5500 Nathan Shock Drive, Baltimore, MD 21224.

Received March 3, 1999; revised August 30, 1999; accepted September 9, 1999. transferase (HPRT), one of the major enzymes of the salvage pathway of purine synthesis. Although HPRT activity is found in all tissues, highest levels are found in the brain and in the basal ganglia in particular. The human HPRT gene lies on the X chromosome (Xq26-27) and contains nine exons distributed over $40 \mathrm{~kb}$ of DNA. The majority of the genetic lesions are point mutations, which affect the ability of the enzyme to catalyze the conversion of hypoxanthine and guanine to their respective nucleotides, inosinic acid and guanylic acid. In the absence of HPRT activity, de novo purine synthesis is increased, leading to the overproduction of uric acid. The clinical presentation includes hyperuricemia and a neuropsychiatric syndrome of choreoathetosis, dystonia, and compulsive aggression against self and others 
(Lesch and Nyhan 1964; Rossiter and Caskey 1994). Despite extensive knowledge on the genetic and biochemical abnormalities associated with LN disease, the mechanisms by which the lack of HPRT produces the typical neuropsychiatric syndrome of LN disease remain unclear.

Although studies of brain function based on postmortem examination, functional neuroimaging, and animal models of LN disease (Goldstein 1986; Breese 1984; Dunnett et al. 1989; Jinnah et al. 1994; Ernst et al. 1996; Wong et al. 1996) have found abnormalities in the dopaminergic system and not in the adrenergic and noradrenergic systems in LN disease, the contribution of the latter cannot be ruled out. The proposition of the adrenergic and noradrenergic involvement in LN disease is based on three types of evidence. The first evidence refers to reports of peripheral noradrenergic dysfunction in subjects with LN disease where unreactive levels of NE to postural stress have been described (Lake and Ziegler 1977). Secondly, the observed behavioral hyperresponsivity and deficit in attention (Matthews et al. 1995) in LN individuals suggests a dysfunction of the arousal system, which is highly dependent on the integrity of the noradrenergic neurotransmitter system. Lastly, measures of aggression have been associated with levels of adrenergic and noradrenergic tone (Coccaro 1996; Gerra et al. 1996; Haller et al. 1998). Relevant to the catecholaminergic neurotransmitter system, low MAO platelet levels have also been reported in LN disease (Breakefield et al. 1976). No studies have yet reported on epinephrine activity in this disorder. The purpose of this study was to assess peripheral adrenergic, noradrenergic, and MAO indices in LN disease.

\section{METHODS}

This study was approved by the Human Subjects Protection Committee of the National Institute of Mental Health, and written informed consent and oral assent were obtained from all parents and subjects, respectively.

\section{Sample}

The sample consisted of nine healthy subjects (eight males and one female; age $22.78 \pm 9.52$ years, 13 to 41 years) and 11 patients with Lesch-Nyhan Disease (11 males; age $14.60 \pm 3.95$ years, 10 to 20 years) who were part of a positron emission tomography study whose findings are presented elsewhere (Ernst et al. 1996).

Control subjects were evaluated by physical examination, routine laboratory tests, and structured psychiatric interview (DICA-Adolescent and DICA-Parent for DSM-III-R diagnoses for adolescents; Schedule for Affective Disorders and Schizophrenia, life-long version for adults) (Reich and Welner 1988; Endicott and
Spitzer 1978). Level of IQ was assessed using the Wechsler adult intelligence scale-revised (WAIS) (Wechsler 1981) and the Wechsler intelligence scale for children revised (WISC-R) (Wechsler 1974) in control adults and children. In both groups, socioeconomic status was evaluated using the Hollingshead rating scale (Hollingshead and Redlich 1958). Exclusion criteria included acute or chronic medical illnesses and DSM-III-R Axis-I psychiatric disorders (American Psychiatric Association 1987).

Subjects with LN disease, referred to our study from around the United States, had been tested for HPRT levels in erythrocytes or fibroblasts at the University of California, San Diego, and Baylor Medical School, Houston Texas. Each patient had documented HPRT levels less than 1 percent of control values. In general, most patients with LN disease are treated with allopurinol, which blocks the production of uric acid. This treatment was not discontinued for the study because there is no evidence that allopurinol interferes with monoaminergic function. In addition, two patients continued their treatment with a benzodiazepine, which they used to reduce self-injury and muscular spasticity. Three others were treated with benzodiazepine, but had not received benzodiazepine for several days prior to the study. Exclusion criteria were medical problems, including chronic seizure disorder, and history of head trauma with loss of consciousness. One boy, included in the study, had had a single seizure several years prior to the study.

All patients presented the neuropsychiatric syndrome typical of LN disease (Anderson et al. 1992; Anderson and Ernst 1994) and were wheelchair-bound in either full or partial restraint one hundred percent of the time. An estimate of IQ was provided using the Stanford Binet Intelligence Scale (Thorndike et al. 1986). All patients and controls had an MRI scan that was read as clinically normal by a neuroradiologist.

\section{Procedure}

All subjects were asked not to eat or drink after midnight prior to the study. Plasma samples were collected in the morning, from an antecubital venous catheter, after subjects had remained seated for a minimum of 20 minutes. The LN subjects were not moved from their wheelchair. Forty-five minutes after applying a topical anesthetic cream (Marcaine Hydrochloride) to the site of puncture, an intravenous catheter with saline drip was placed in the antecubital vein. A 10-cc blood sample was drawn within the next hour through the IV catheter into tubes containing EDTA, and was stored at $-80^{\circ} \mathrm{C}$ until assayed. Although anxiety level was not rated, the LN subjects appeared generally more anxious and agitated than the control subjects during the blood drawing. Baseline vital signs were measured on admis- 
sion, prior to the study, but not at the time of blood sampling.

Plasma concentration of the monoamines and their metabolites were estimated by high performance liquid chromatography with electrochemical detection. A preextraction procedure using alumina was employed for the catecholamines (Eisenhofer 1986; Murphy 1991).

\section{Analysis of the Results}

A multivariate analysis of variance (MANOVA) compared NE, EPI, and DHPG measures between the LN group and the control group. Because controls and LN subjects differed in mean age, an exploratory analysis was performed to assess age as a potential covariate for catecholamine blood levels. Group status and age were entered into a multiple regression analysis, where the order of entry was based on the strength of the univariate correlations, and the threshold for entry was set at $p<.25$. Age did not produce a significant increase in the amount of variance accounted for above group membership in any of the catecholamine levels and was thus not used as a covariate. In addition, age was not significantly correlated with measures of EPI, NE and DHPG in neither control nor LN group. Correlations of the biochemical measures (that significantly differed between groups) with $I Q$, an estimate of severity of illness, were tested in each group separately using Pearson coefficient correlation analyses.

\section{RESULTS}

\section{Sample}

One LN subject was removed from the analysis of the data because his DHPG plasma concentration was seven standard deviations higher than the mean of the DHPG plasma concentration in the LN group. Although this subject was one of the two boys on benzodiazepine treatment, the other boy had plasma catecholaminergic concentrations within one standard deviation of the group means. The female included in the control group had plasma levels values also within one standard deviation of the control group means, and was kept in the analysis of the results.

The LN group was significantly younger than the control group, but similar with respect to socioeconomic status (Table 1). As already mentioned in the Methods, age did not influence group differences in plasma catecholamine concentrations. As expected, IQ was significantly lower in the LN group compared to the controls. Baseline vital signs showed no statistical differences in systolic or diastolic blood pressures. Pulse rate was significantly higher in the LN patients than in the control subjects.
Table 1. Characteristics (Mean \pm SD) of the Control $(n=9)$ and Lesch-Nyhan $(n=10)$ Group

\begin{tabular}{lccl}
\hline & Control & Lesch-Nyhan & $\boldsymbol{P}$ \\
\hline AGE & $22.78 \pm 9.52$ & $14.60 \pm 3.95$ & .04 \\
SES & $55.39 \pm 37.34$ & $59.20 \pm 20.81$ & NS \\
IQ & $119.14 \pm 14.51$ & $67.90 \pm 17.76$ & .000 \\
Systolic & $119.67 \pm 7.83$ & $117.90 \pm 10.99$ & NS \\
Diastolic & $77.56 \pm 6.02$ & $74.0 \pm 12.99$ & NS \\
Pulse & $70.56 \pm 11.59$ & $90.78 \pm 15.06$ & .006 \\
\hline
\end{tabular}

NS, non significant.

\section{Plasma Catecholaminergic Concentrations}

The overall MANOVA with group as between subjects factor and plasma concentrations of NE, EPI, DHPG as within subjects factors was highly statistically significant (Wilks Lambda $=0.05, \mathrm{~F}[2,16]=152.9, p=.000$ ) (Table 2). Post-hoc analysis showed that the LN group had significantly higher EPI concentrations by $245 \%$ $(\mathrm{F}[1,18]=19.3, p=.000)$ (Figure 1), and lower DHPG concentrations by $42 \%(F[1,18]=34.3, p=.000)$ (Figure 2$)$ compared to the control group. Levels of DHPG were not correlated with levels of EPI in either group. Levels of NE did not differ significantly between groups. Results were similar when the outlier LN subject was included in the analysis (data not shown here).

\section{Associations between Vital Signs and Catecholaminergic Blood Levels}

No associations were noted between DHPG or EPI levels and baseline systolic pressure, diastolic pressure, and pulse rate in either control or LN group.

\section{Associations between Behavioral Variables and Catecholaminergic Blood Levels}

Pearson coefficient correlations showed that IQ was significantly associated with EPI ( $\mathrm{r}=0.77 ; p=.009)$ (see Figure 3), and not with DHPG levels. In the control group, IQ was not correlated with EPI or DHPG measures.

Table 2. Catecholaminergic Blood Levels (pmol/ml) (Mean \pm SD) in the Control $(n=9)$ and Lesch-Nyhan $(n=10)$ Groups

\begin{tabular}{lccc}
\hline & Control & Lesch-Nyhan & $\boldsymbol{P}$ \\
\hline EPI & $35.89 \pm 31.33$ & $123.80 \pm 52.03$ & .00 \\
NE & $309.78 \pm 188.71$ & $232.78 \pm 154.96$ & NS \\
DHPG & $757.56 \pm 146.09$ & $440.30 \pm 85.28$ & .00 \\
\hline
\end{tabular}

NS, non significant. 


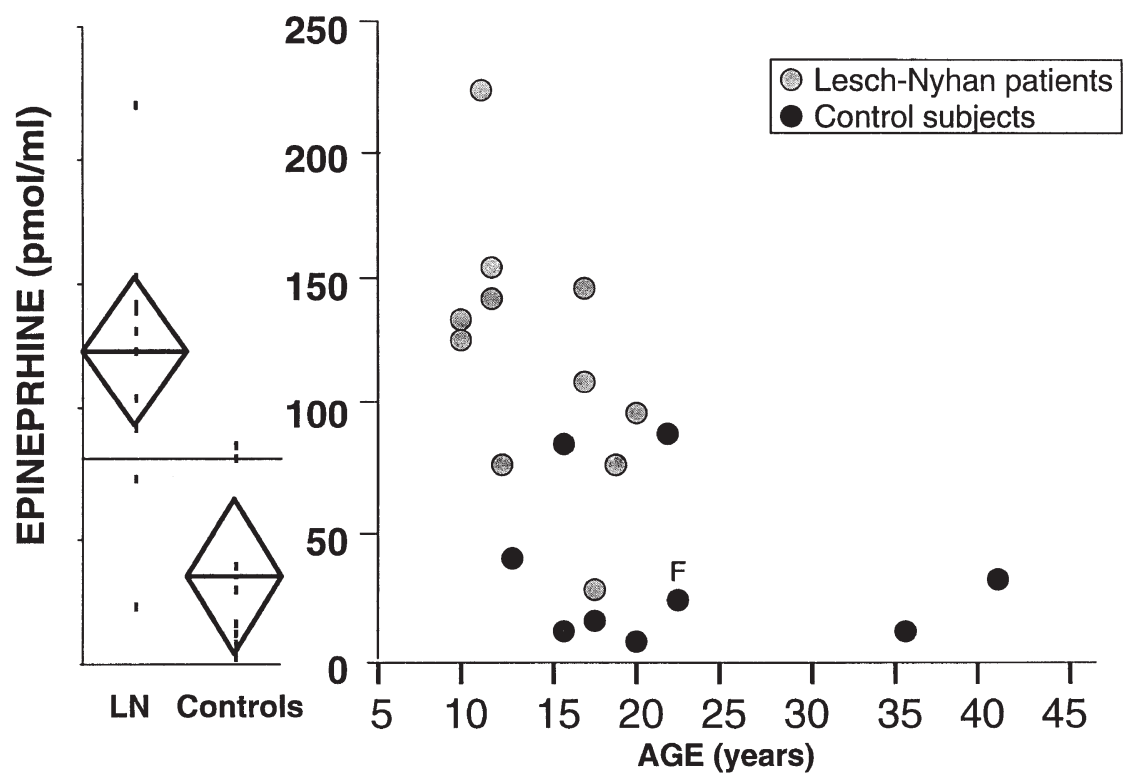

Figure 1. Scatter plot of individual values of epinephrine plasma concentrations $(\mathrm{pmol} / \mathrm{ml})$ in Lesch-Nyhan patients $(n=10)$ and healthy controls $(n=9)$ as a function of age. The horizontal line in each diamond represents the group mean, its length the group size, and its height the $95 \%$ confidence interval. The female control is indicated by an " $F$ ".

\section{DISCUSSION}

The LN group had significantly higher EPI and lower DHPG plasma concentrations than the control group. No group differences were noted in NE plasma levels.

Some caveats need to be addressed before discussing the findings. They include a small sample size and large variability of the dependent variables. Also, the difference in age between the two groups may have introduced additional variability. However, the presentation of these findings is justified by the uniqueness of the data, and their potential use for the development of pathophysiological models that can be tested in hypotheses-driven studies.

The interpretation of the EPI findings is limited by the lack of measures of the degree of arousal and stress in the LN subjects. Indeed, the elevation of EPI plasma levels due to psychological stress is well established (Glavin 1983; Stone 1983a,b; Finlay 1995). However, such elevation does not commonly exceed $200 \%$ of basal values in humans. For example, increases of epinephrine plasma levels in healthy adults were shown to reach $59 \%$ and $54 \%$ of basal levels following laboratoryinduced and real life stress, respectively (van Doornen 1992). The chronic stress that characterizes the lives of LN individuals (e.g., threat of hurting themselves or others when not physically restrained) may result in chronic abnormal adrenergic/noradrenergic function.

Two sets of data suggest that the excess of psychological stress that LN subjects may have experienced relative to control subjects during the experimental procedure was not the only determinant in the abnormally

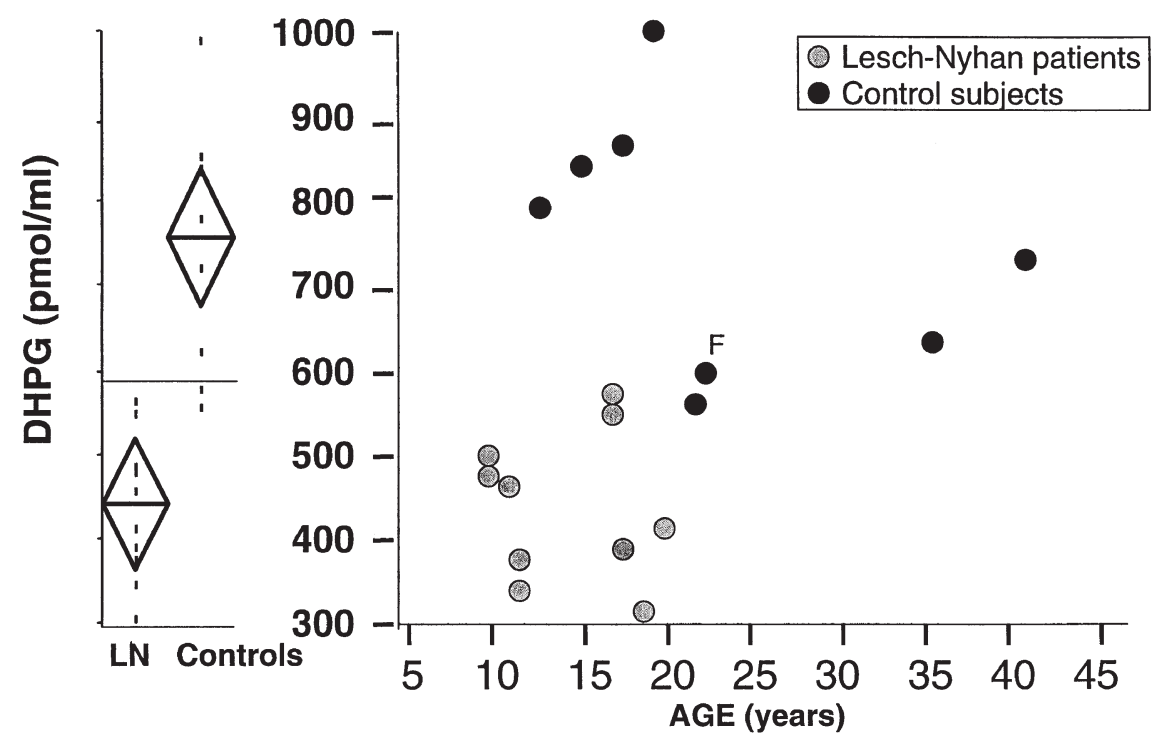

Figure 2. 3-methoxy-4-hydroxyphenylglycol (DHPG) $(\mathrm{pmol} / \mathrm{ml})$ plasma levels in Lesch-Nyhan patients $(n=$ $10)$ and healthy controls $(n=9)$ as a function of age. The horizontal line in each diamond represents the group mean, its length the group size, and its height the $95 \%$ confidence interval. The female control is indicated by an "F". 


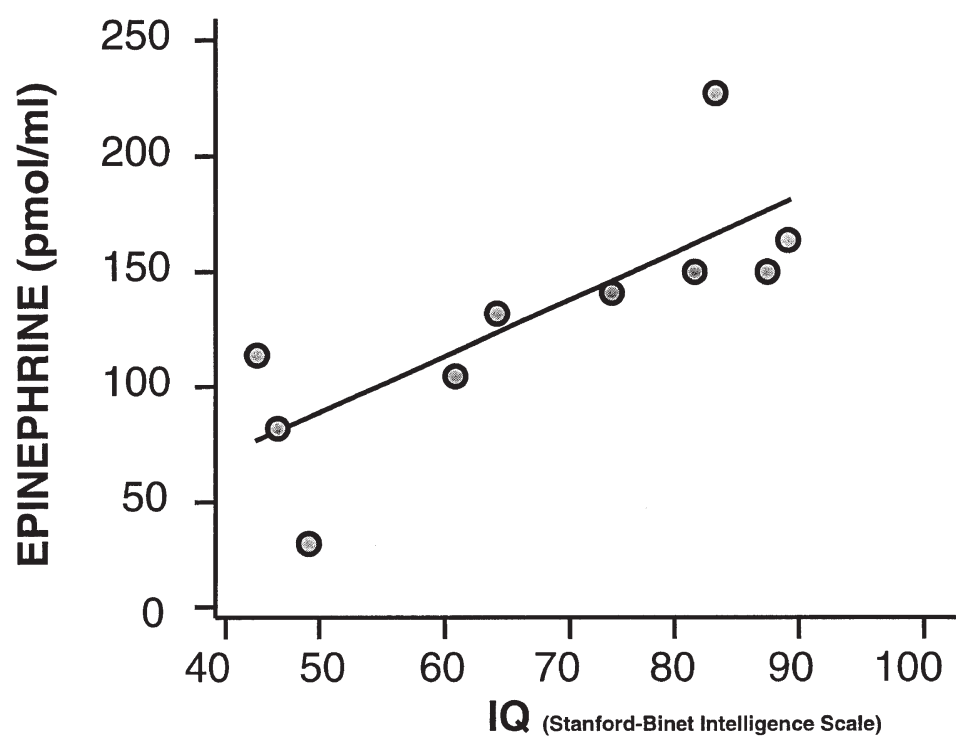

Figure 3. Correlation between epinephrine plasma concentrations $(\mathrm{pmol} / \mathrm{ml})$ and IQ in the Lesch-Nyhan group $(n=10)$. high EPI levels in these patients. First, the exclusion from the analysis of the two LN subjects who showed the worst reaction to the catheter placement (psychomotor agitation, screaming), even though it occurred at least 20 minutes prior to the blood sampling, did not alter the statistical significance of the differences in EPI levels between the groups. Second, the noradrenergic system (NE, DHPG) would be expected to be also activated, albeit not to the same degree as the adrenergic system, if stress were responsible for sympathetic activation (Elwood 1986).

Elevated EPI plasma concentrations in the face of unchanged NE levels may reflect a selective biochemical deviance that affects the adrenal medulla (which is the main origin of EPI in the periphery) or its regulation by the hypothalamo-pituitary axis. Alteration of this axis is conceivable in view of the growth retardation and delay of sexual maturation observed in LN subjects (Rossiter and Caskey 1994). This sexual maturation delay was evident in our LN sample, where all three 12-yearold LN boys were still in Tanner stage 1 (range 1-5), two of the three 17 year-old boys in stage 2, and the third of the 17 year-old in stage 4 . A partial defect in the adrenocortical 11 beta-hydroxylation of steroids has been demonstrated after ACTH stimulation in four LN patients (Watts 1987). However, thyroid function, which would affect adrenal function, was normal in all subjects of this study (assessed by blood thyroid screen).

Notwithstanding, EPI concentrations have not been assessed in any of the published biochemical studies of LN disease (Rockson 1974; Lake and Ziegler 1977; Silverstein et al. 1985; Jankovic et al. 1988). In contrast, NE levels have been reported to be normal or reduced at baseline, and irresponsive to postural changes (Lake and Ziegler 1977; Rockson 1974), or to exposure to cold (Rockson 1974).
Findings of normal NE and low DHPG support reduced noradrenergic function. Consistent with this hypothesis is the observation of normal blood pressure in the face of a chronic hyperarousal state. Indeed, LN subjects are characterized by a state of hyperexcitability. For example, the LN individual tends to react with an outburst of choreoathetoid movements when verbal or even only visual contact is initiated with him in the course of an interview with his family. In this context of hyperarousal (or hyperarousability), the absence of relatively elevated blood pressure may actually reflect a reduced function of the sympathetic system, as does the absence of noradrenergic response to postural changes. In addition, the elevated pulse rate in LN subjects compared to controls may reflect the higher adrenergic tone rather than the influence of the noradrenergic function, or the differences in age or in cardiovascular fitness between groups. The abnormally low DHPG levels may thus represent an adaptive mechanism to spare NE levels, possibly mediated by reduced MAO activity. This finding is consistent with the report of low CSF MHPG in 5 LN boys (Jankovic et al. 1988), and low to normal plasma levels of NE in $14 \mathrm{LN}$ subjects compared to controls (Lake and Ziegler 1977). Of interest, central noradrenergic function was reported to be normal in postmortem biochemical analyses of NE concentration (Lloyd et al. 1981), and in a genetic mouse model of LN (Jinnah et al. 1994).

In conclusion, peripheral catecholaminergic measures showed a dissociation of peripheral indices of noradrenergic and adrenergic measures, where adrenergic tone is enhanced whereas noradrenergic function is either reduced or compensated by decreased catabolism of NE, probably mediated by MAO hypoactivity. Given the evidence of abnormal adrenergic function peripherally, it would be of great interest to explore this 
system centrally. In addition, MAO function may also play an important role in the cerebral dopaminergic deficit that has been identified in previous work (Lloyd et al., 1981; Ernst et al., 1996; Wong et al., 1996) in LN subjects. Based on the present findings, we suggest that a reduction of MAO activity peripherally could be an adaptive mechanism for the sparing of NE concentrations. Such compensatory mechanism could also operate centrally. However, the clinical consequences of this adaptive mechanism is unclear. Indeed, the contribution to the aggressive behavior of the putative reduced MAO activity in LN disease is to be considered, based on the report of unusual aggressiveness associated with the lack of MAO-A in human males (Brunner et al. 1993) and in transgenic male mice (Cases et al. 1995). Finally, the high adrenergic tone may play a role in some of the accompanying symptoms of LN such as dysphagia, which is a severe problem for many subjects with LN disease, since EPI is a neurotransmitter active in smooth muscles.

\section{ACKNOWLEDGMENTS}

We thank Dr. James Hankerson, Peter Jons, Akia Talbot, Teresa Talliner, and Lars Hungerford for their assistance in performing the study.

\section{REFERENCES}

American Psychiatric Association (1987): Diagnostic and statistical manual of mental disorders. 3rd ed (revised). Washington, DC, American Psychiatric Association Press

Anderson LT, Ernst M (1994): Self-injury in Lesch-Nyhan Disease. J Autism Dev Disord 24:67-81

Anderson LT, Ernst M, Davis SV (1992): Cognitive abilities of patients with Lesch-Nyhan Disease. J Autism Dev Disord 22:189-203

Breakefield XO, Castiglione CM, Edelstein SB (1976): Monoamine oxidase activity decreased in cells lacking hypoxanthine phosphoribosyltransferase activity. Science 192:1018-1020

Breese GR (1984): Neonatal-6-hydroxydopamine treatment: Model of susceptibility for self-mutilation in the LeschNyhan syndrome. Pharmacol Biochem Behav 21:459-461

Brunner H, Nelen M, Breakfield X, Ropers H, Van Oost A (1993): Abnormal behavior associated with a point mutation in the structural gene for monamine oxidase A. Science 22:578-580

Cases O, Seif I, Grimsby J, Gaspar P, Chen K, Pournin S, Muller U, Aguet M, Babinet C, Shih JC, De Maeyer E (1995): Aggressive behavior and altered amounts of brain serotonin and norepinephrine in mice lacking MAOA. Science 268:1763-1766

Coccaro EF (1996): Neurotransmitter correlates of impulsive aggression in humans. Ann NY Acad Sci 20(794):82-89
Dunnett SB, Sirinathsinghji DJ, Heavens R, Rogers DC, Kuehn MR (1989): Monoamine deficiency in a transgenic (Hprt-) mouse model of Lesch-Nyhan syndrome. Brain Res 501:401-406

Eisenhofer G (1986): Simultaneous liquid-chromatographic determination of 3,4-dihydroxyphenylglycol, catecholamines, and 3,4-dihydroxyphenylalanine in plasma, and their responses to inhibition of monoamine oxidase. Clin Chem 32:2030-2033

Elwood SW (1986): Catecholamine response of children in a naturally occurring stressor situation. J Human Stress 12:154-161

Endicott J, Spitzer RL (1978): A diagnostic interview: The schedule for affective disorders and schizophrenia. Arch Gen Psychiatry 35:837-844

Ernst M, Zametkin AJ, Matochik JA, Pascualvaca D, Jons PH, Hardy K, Hankerson JG, Doudet DD, Cohen RM (1996): Presynaptic dopaminergic deficits in LeschNyhan Disease. N Engl J Med 334:1568-1604

Finlay JM (1995): Increased dopamine and norepinephrine release in medial prefrontal cortex induced by acute and chronic stress: Effects of diazepam. Neuroscience 64:619-628

Gerra G, Avanzini P, Zaimovic A, Fertonani G, Caccavari R, Delsignore R, Gardini F, Talarico E, Lecchini R, Maestri D, Brambilla F (1996): Neurotransmitter and endocrine modulation of aggressive behavior and its components in normal humans. Behav Brain Res 81:19-24

Glavin GB (1983): Effects of altered brain noradrenaline level on acute stress pathology in rats. Kurume Med J 30:31-34

Goldstein M (1986): Dopamine agonist induced self-mutilative biting behavior in monkeys with unilateral ventromedial tegmental lesions of the brainstem: Possible pharmacological model for Lesch-Nyhan syndrome. Brain Res 367:114-120

Haller J, Makara GB, Kruk MR (1998): Catecholaminergic involvement in the control of aggression: Hormones, the peripheral sympathetic, and central noradrenergic systems. Neurosci Biobehav Rev 22:85-97

Hollingshead AB, Redlich F (1958): The two-factor index of social position. In Social Class and Mental Illness. New York, NY, Wiley

Jankovic J, Caskey TC, Stout T, Butler IJ (1988): Lesch-Nyhan syndrome: A study of motor behavior and cerebrospinal fluid neurotransmitters. Ann Neurol 23:466-469

Jinnah HA, Wojcik BE, Hunt M, Narang N, Lee KY, Goldstein M, Wamsley JK, Langlais PJ, Friedmann T (1994): Dopamine deficiency in a genetic mouse model of Lesch-Nyhan disease. J Neurosci 14:1164-1175

Lake CR, Ziegler MG (1977): Lesch-Nyhan syndrome: Low dopamine- $\beta$-hydroxylase activity and diminished sympathetic response to stress and posture. Science 196:905-906

Lesch M, Nyhan WL (1964): A familial disorder of uric acid metabolism and central nervous system function. Am J Med 36:561-570

Lloyd KG, Hornykiewicz O, Davidson L, Shannak K, Farley I, Goldstein M, Shibuya M, Kelley WN, Fox IH (1981): Biochemical evidence of dysfunction of brain neurotransmitters in the Lesch-Nyhan syndrome. N Engl J Med 305:1106-1111 
Matthews WS, Solan A, Barabas G (1995): Cognitive functioning in Lesch-Nyhan syndrome. Dev Med Child Neurol 37:715-722

Murphy DL (1991): Plasma amine oxidase activities in Norrie disease patients with an X-chromosomal deletion affecting monoamine oxidase. J Neural Transm Gen Sect $83: 1-12$

Reich W, Welner Z (1988): Diagnostic Interview for Children, Adolescents, and Parents. St. Louis, MO, Washington University

Rockson S (1974): Lesch-Nyhan syndrome: evidence for abnormal adrenergic function. Science 186:934-935

Rossiter BJF, Caskey CT (1994): Hypoxanthine-guanine phosphoribosyltransferase deficiency: Lesch-Nyhan syndrome and gout. In Scriver CR, Beaudet AL, Sly WS, Valle D (eds), The Metabolic and Molecular Bases of Inherited Disease. 7th ed. New York, McGraw-Hill, pp 1679-1706

Silverstein FS, Johnston MV, Hutchinson RJ, Edwards L (1985): Lesch-Nyhan syndrome: CSF neurotransmitter abnormalities. Neurology 35:907-911

Stone EA (1983a): Adaptation to stress and brain noradrenergic receptors. Neurosci Biobehav Rev 7:503-509
Stone EA (1983b): Adaptation to stress: Tyrosine hydroxylase activity and catecholamine release. Neurosci Biobehav Rev 7:29-34

Thorndike, RL, Hagen, EP, Sattler, JM (1986): The StanfordBinet Intelligence Scale. 4th ed.. Riverside, Chicago Publishing Company

van Doornen LJ (1992): The relationship between cardiovascular and catecholamine reactions to laboratory and real-life stress. Psychophysiology 29:173-181

Watts RW (1987): Lesch-Nyhan syndrome: Growth delay, testicular atrophy and a partial failure of the 11 betahydroxylation of steroids. J Inherit Metab Dis 10:210-223

Wechsler D (1981): WAIS-R: Wechsler Adult Intelligence Scale-Revised. New York: New York, NY, The Psychological Corporation

Wechsler D (1974): WISC-R Manual: Wechsler Intelligence Scale for Children-Revised. New York, NY, The Psychological Corporation

Wong DF, Harris JC, Naidu S, Yokoi F, Marenco S, Dannals RF, Ravert HT, Yaster M, Evans A, Rousset O, Bryan RN, Gjedde A, Kuhar MJ, Breese GR (1996): Dopamine transporters are markedly reduced in Lesch-Nyhan disease in vivo. Proc Natl Acad Sci USA 93:5539-5543 